\title{
Recent Developments on Algal Biofuel Technology
}

Rachel Long, Philip Sanderson and Pattanathu K.S.M. Rahman*

Technology Futures Institute, School of Science and Engineering,

Teesside University, Tees Valley - TS13BA, United Kingdom

(Corresponding author: p.rahman@tees.ac.uk)

\section{Abstract:}

Microalgae provide great promise for the production of biofuels as they have the highest growth rates of all photosynthetic organisms and have demonstrated high levels of desirable products required for biofuel production without competing for land or resources from the agriculture industry. It has been established that the type of algae chosen to cultivate is very important. This can effect which techniques may be best to use for harvesting and converting algae to biofuels. It is important to consider the lipid content of the algae and its growth rate as a high yield shall be needed from the process, but it must also be of high quality. The research that has been conducted so far has proven very useful however there is still much to learn when it comes to the best techniques to use for the production of biofuels. Any process utilizing microalgae needs to be cost effective to be commercially viable and be able to fit into the current 
fuel demand without altering distribution or storage processes. In order to achieve a positive energy balance which will be important when considering cost effective processes, a highly optimized production system is required. In recent scientific discussions, many of the environmental impacts and cost performance points raised produce both challenges and opportunities that would require further research and the potential integration of other processes. Once a gold standard has been established for the cultivation of algal biomass and lipid extraction methods, commercialization of algal biofuel production will soon follow.

\section{Introduction:}

There have been many new developments in the field of biotechnology over the past 5 years, and marine biotechnology has been one of the most researched disciplines since it was recognized in the 1980s (Burgess, 2012). Marine biotechnology is a discipline where resources come from the ocean habitats rather than land habitats. The ocean has a high biological and chemical diversity; this is due to the ocean having so many unique habitats and has existed for so many more years than terrestrial habitats. Recent advances such as whole genome sequencing and metagenomics are revealing greater biodiversity than previously thought in the marine environment, diversity that remains largely untapped (Ritchie et al., 2013). As a result of extreme environmental conditions such as temperature, light and the change in oxygen concentration, it has aided in the adaptation of many organisms with unique features where new bioactive compounds have been created. 
Biofuel is a renewable energy source produced from biomass which can be used as a substitute for petroleum fuels (Demirbas, 2010). The ever growing requirement for biofuels is somewhat demanding, relying on the ability for them to be able to fit into the existing infrastructure for storage and distribution on a huge scale without competing with food crops for land or the food industry for biomass (Leite, et al., 2013; Li et al., 2013). Due to the growing concern associated with the depletion of nonrenewable fuel sources many sources of biomass have been proposed as biofuel feedstock, these include vegetable oil, jatropha and microalgae (Perez-Garcia et al., 2011; Suali and Sarbatly, 2012). This depletion has increased in recent years due to rapid development growth in newly developing countries and also higher consumption rates of currently utilized fuels due to an increase in industrial growth (Adenle, et al., 2013). This suggests that we need to find a way of creating these fuel types manually, but also create them in a way that is environmentally friendly and reproducible on a commercial scale.

It seems like a strange idea as microalgae is thought of as an aquatic photosynthetic organism, but it is known that Microalgae with the simple composition $\mathrm{CH}_{1.7} \mathrm{O}_{0.4} \mathrm{~N}_{0.15} \mathrm{P}_{0.0094}$, are able to convert $\mathrm{CO}_{2}$ and $\mathrm{H}_{2} \mathrm{O}$ into biomass using sunlight (Demirbas, 2010; Zhu et al., 2014). Algae have shown the ability to synthesis and accumulate a variety of high energy molecules including fatty acids and triacylglycerols. This accumulation has shown a lipid content of up to $73.4 \%$ which mostly consists of these high energy molecules, which can be converted through the process of transesterification into biodiesel (Suali and Sarbatly 2012; Leite et al., 2013). It has also been found that some microalgal species can double their biomass in a short a time as three and a half hours, Microalgae has a higher growth rate than 
terrestrial plants and require less water in order to grow, in addition to this, microalgae can potentially be produced in an economical and sustainable manner (Demirbas, 2010; Lam and lee, 2012; Suali and Sarbatly, 2012)

There are several main hurdles facing the economical and sustainable production of algal biofuel; cultivation, lipid yield, extraction, purification and cost. Lam and Lee (2012), critically discussed the concerns and limitations of processing of microalgae for the production of biofuels and the potential way forward. The discussion focused around 2 alternatives for the cultivation of photoautotrophic algae; open pond systems and photo-bioreactors (PBR's). Environmental concerns that are highlighted within the paper are also described to have positive potentials if the processes are integrated with other industrial processes such as water remediation, heat and $\mathrm{CO}_{2}$ from power generation, toxicological, chemical and biomedical research. One concern that is raised is that leakage and transfer of genetically modified algae or natural algae into the environment is unavoidable (Lam and Lee, 2012). Fuels have already been developed from the fermentation and esterification of some crops in the USA and some European countries (Adenle et al., 2013). This type of fuel is known as first generation biofuels, however they are not entirely supported as they create the argument for resources between food and fuel. This makes algae the perfect option as we know they can produce high lipid yield so we can potentially create a large amount of biofuel without depleting or competing with any food crops. We could continue to use crops as a fuel source, but in order to do so, vast increases in the amount of crops grown would need to be seen in order to cover the consumption of these crops in both the food and the fuel industry. This would be incredibly expensive due to the amount of man power and land that would be needed. An additional problem 
with crops being used as a fuel source is that the competition for materials between the two markets with force the prices to rise for consumers.

Microalgae have been promoted as one of the most promising third-generation biofuels for their ability to create such a large amount of lipid, they grow in low quality water and divide at a rapid pace (Rogers, 2013). They have been found to be more efficient in converting sunlight to biochemical energy than terrestrial plants and green microalgae are known to have the same photosynthetic machinery as C3 plants, they can convert $4.6 \%$ of solar energy that they receive into biomass energy and have been known to reach up to $8.3 \%$ conversion (Chisti, 2013).

\section{Algal Growth}

There are several different methods of microalgae lipid conversion to biofuel, but the first thing to consider is the growth of the microalgae and its lipid yield. There are two ways in which microalgae grow, through phototrophic growth where the algae uses light and $\mathrm{CO}_{2}$ and releases $\mathrm{O}_{2}$ through the process of photosynthesis, for this process the algae needs a light environment with plenty of $\mathrm{CO}_{2}$, however the growth of the algae is slow and their lipid content tends to be low. Microalgae can also grow through the use of heterotrophic growth where they use sugars and $\mathrm{O}_{2}$ and release $\mathrm{CO}_{2}$ through the use of respiration, the algae need a dark environment so that phototrophic growth can be prevented. This type of growth tends to produce high lipid content in the algae and a fast growth rate (Figure 1).

There are several culturing methods to consider for the growth of algae depending on the type of growth that is decided upon. Raceway ponds and Circular ponds are the two main types of open pond currently used (Leite et al., 2013), they are a simple and 
cheap way of culturing algae. Open pond cultivation has been in use since the 1950's (Christenson and Sims, 2011) and are the oldest and simplest methods of mass cultivation of microalgae (Demirbas, 2010), however only a select few species can be cultured in conventional open systems as the control of physio-chemical conditions is limited (Silva Benavides et al, 2013). Open pond cultivation is carried out in shallow basins in order to prevent self-shadowing, the ponds are also open to the environment and this can present potential problems due to environmental contamination, contamination with unwanted algal species and inefficient use of $\mathrm{CO}_{2}$ due to evaporation (Wen et al., 2011). Open ponds are equipped with a device which creates hydrodynamic conditions to mix algal cells along a light path (Hadiyanto et al, 2013). Sun light is used as the main energy source for photosynthesis while $\mathrm{CO}_{2}$ is injected into the pond system.

\subsection{Open Ponds}

Raceways are one open pond method of algae cultivation, these ponds are closed loop systems which are typically quite shallow to ensure that light penetrates all levels of the pond. In these ponds paddle wheels are used to mix the algae constantly; one paddle wheel is enough to mix algae in raceways covering very large areas, this maintains aeration and nutrient dispersion with low cost and low energy consumption whilst preventing sedimentation (Chisti, 2007; Joquera et al., 2010; Leite et al., 2013). As the cultivation of algae highly depends on large volumes of water a large amount of land and water are needed. Lack of water due to evaporation can cause major sustainability issues if the process was to be scaled up (Rogers, 2013), although evaporation of the water is essential in cooling to keep the appropriate pond temperature. Here is where covered raceways can be used as any evaporated water 
could be collected and then re-deposited into the raceways. Covered raceways have been found to support 4.2 times the production capacity compared to uncovered raceways. Most algal raceways use channels which have a constant depth and width, which end in hairpin bends with one or more paddle wheels to circulate the water (Liffman, 2013).

Circular open ponds work in very much the same way as raceway ponds but the pond is entirely circular and mixing is maintained by a paddle which is fixed at the center of the pond to the perimeter edge and rotates slowly. In comparison to raceway ponds, circular open ponds have poor mixing and light distribution properties (Suali and Sarbatly, 2012).

\subsection{Photo-bioreactors (PBR's)}

Photo-bioreactors tend to be a mass of connected suspended compartments which contain algae and water and stirred by mechanical stirring and/or bubble sparging. This method of cultivation heightens the absorbance as sunlight is reflected off the clear surfaces and back onto the algae (Lee et al, 2014). Photo-bioreactors, unlike open ponds, permit the culture of single-species microalgae and prevent environmental contamination of unwanted algal species and potential contamination of the environment with genetically modified microalgae (Leite et al., 2013). In addition to this they also have a high productivity per unit area and enable the control of $\mathrm{pH}$, temperature, light intensity and nutrient availability (Chisti, 2007; Leite et al., 2013). The most widely used photo-bioreactor design is tubular which has a large surface area to volume ratio to maximize exposure to sunlight and sunlight penetration (Wen et al., 2011). In closed systems like photo-bioreactors, the oxygen produced during 
photosynthesis will build up until it inhibits growth and potentially poisons the algae (Wen et al., 2011; Suali and Sarbatly, 2012). This build-up of oxygen can be alleviated using a degassing zone where air is bubbled through the broth to remove excess oxygen (Chisti, 2007; Wen et al., 2011).

These bioreactors can also be kept in the dark for heterotrophic growth (Figure 2). Varied lipid contents are noted using heterotrophic culture methods in Suali and Sarbatly (2012) ranging from $44.8 \%$ when using glucose as the carbon source, to $73.4 \%$ using different carbon sources such as crude glycerol or sweet sorghum in the culture medium. Despite this high lipid content, heterotrophic microalgae cultivation would not be as cost effective as phototrophic culture when the carbon source has to be purchased. Research has found that Chlorella sp., algae can be manipulated to utilize $\mathrm{CO}_{2}$ (Suali and Sarbatly, 2012). This is seen as a promising source of organic carbon, when carbon dioxide produced by power plants burning fossil fuels is often available at little or no cost (Chisti, 2007). This could provide a suitable alternative as a carbon source using a mono culture under heterotrophic conditions. In addition to this Chlorella spp., especially Chlorella pyrenoidosa, Chlorella ellipsoidea and Chlorella vulgaris have been found to be suitable for further research for microalgae cultivation for biofuel production. This is due to their successful cultivation both in heterotrophic and photoautotrophic conditions whilst still producing a high lipid content (Han et al., 2012).

\section{Separation Techniques}

The collection method of the algae now needs to be determined. There are several harvesting methods used for the separation of the microalgae from the solution but there is currently no gold standard or universal algae harvesting method widely 
available (Mata et al., 2010). Harvesting the algae from the cultivation stage can be very costly, between $20-30 \%$ for the total cost of production as cell concentration is very dilute and therefore a large volume of medium needs to be treated in order to harvest suitable quantities of biomass for lipid extraction (Wen, et al., 2011). Harvesting methods can be chemical, mechanical or to a lesser extent electrical based, examples of these include flocculation, filtration, centrifugation, sedimentation, flotation or ultrasound techniques (Suali and Sarbatly, 2012), variations or sequences of these methods are also commonly used. These methods all discriminate on a size and density basis (Mata et al., 2010) which can hinder specific techniques when using different algae species due to the natural variation in size. Flocculation is not a critical step in separating algal cells from the medium as processes have been described just involving sedimentation, filtration or centrifugation alone (Wen et al., 2011) but the aggregated cells become easier to harvest as flocculent agents lead to the coagulation of the algae (Suali and Sarbatly, 2012). Biological methods are also being researched as a more ecofriendly and cost effective way of harvesting algae.

\subsection{Flocculation}

Chemical flocculation is mostly used as a pretreatment for small algae cells to increase the particle size before using another harvesting method such as flotation or filtration (Christenson and Sims, 2011). The use or potential use of flocculation as part of a biomass harvesting technique is being described in recent research (Christenson and Sims, 2011; Wen et al., 2011; Lam and Lee, 2012; Suali and Sarbatly, 2012; Leite et al., 2013). Ideal Flocculants would have to be low cost, non-toxic and effective at low concentrations in order to be commercially viable due to their high cost (Wen et al., 2011). Its use can speed up or facilitate other separation techniques using a flocculating agent although many of these flocculating agents are described as being 
toxic so prevents the use of the biomass after lipid extraction as animal feed or for other processes (Leite et al., 2013). The use of metal salts for coagulation and flocculation is cautioned when considering a downstream process. Methanogenic activity of acetogenic and methanogenic bacteria fed waste water sludge has been found to be inhibited by aluminum and sulfate. Application of sludge treated by aluminium has been seen to increase heavy metal uptake and cause phosphorus deficiencies in plants, however natural polymers may also be used as flocculants but this had not been as extensively researched (Christenson and Sims, 2011). The efficiency of flocculation depends highly upon the type of algal cells used. The amount of cells flocculated tends to depend on the concentration of the algal cells. Cationic starches are an ecologically friendly and effective alternative for the processing of algae to biofuel (Gerde et al, 2014). More recently, bioflocculants have been described in Kim et al., (2013) as being environmentally friendly and demonstrating successful harvesting of $95 \%$ of biomass, this could potentially overcome the inherent problem of toxic contamination when using traditional chemical flocculation and is certainly something that requires further investigation.

\subsection{Sedimentation}

The use of sedimentation as a harvesting method is very low cost and can typically give concentrations of $1.5 \%$ solids (Christenson and Sims, 2011), but reliability is low due to the fluctuation in algal cell sizes, it is also a slow process as the algal cells are left to drift to the bottom of the culturing media naturally, this could affect the quality of the algae if it is left for a period of time. Sedimentation is achieved by some algae strains that have the property of natural sedimentation in the absence of mixing, the capital and operational costs of this harvesting method are low but the process can be time consuming (Wen, et al., 2011; Leite et al., 2013). The sedimentation process can 
be sped up and increased in algae with low sedimentation properties by the addition of a flocculation agent (Wen et al., 2011).

\subsection{Centrifugation}

The most reliable and quick method of harvesting algae is possibly the use of a centrifuge, which separates the suspended algae due to the density of the mixture. Centrifuges with nozzle type disks are easily cleaned and sterilized, making them ideal for any type of algae separation. The samples are kept pure and uncontaminated ensuring the end product will remain of good quality. However this form of harvesting is very time consuming and expensive, making it an unrealistic permanent harvesting method. The process also involves exposing cells to high gravitational forces which can damage the cell structure (Knuckey et al., 2006). Centrifugation provides an effective separation of biomass from the medium within a short time but incurs a high

cost due to the initial startup costs and power consumption (Kim et al., 2013). This harvesting technique is currently used for the recovery of high value products to avoid bacterial contamination or fouling of the raw product but it is claimed that it would not be cost effective for the recovery of biomass for biofuels due to their lower value (Mata et al., 2010, Kim et al., 2013). It has, however, been discussed that if the volumetric throughput was increased there is potential for it to become more cost effective but with lower efficiency (Leite et al., 2013).

\subsection{Filtration}

In past studies low-cost filtration has been used to harvest filamentous algae strains at a high rate algal pond (Christenson and Sims, 2011). Micro screening is used to retain larger cells and wash out smaller cells of algae that are non-filamentous. However the use of filamentous algae has been found to be unrealistic as they have 
a low lipid content, for algae of a smaller size the method that is considered most reliable is that of tangential filtration. Filtration is currently widely used in biotechnology for extraction of products from liquid medium due to the lack of requirement for chemicals, its continuous operation and its high separation efficiency. It also enables the recycling of the culture medium which may contain valuable nutrients for reuse (Kim et al., 2013). Most microalgae are too small for filtration (Leite et al., 2013) and those that are small cause plugging of the filter which is a major limitation for filtration (Wen et al, 2011). Plugging can be overcome by backwashing to maintain the efficiency of the membrane but this is time consuming (Suali and Sarbatly, 2012). A lot of filtration processes have been increasing in cost due to the need for pumping, backwashing, additional steps and possible replacement of membranes on a commercial scale (Mata et al., 2010).

\subsection{Ultrasound}

Ultrasound presents the possibility of both algal harvest and lipid extraction (Kim et al., 2013) although further research needs to be completed in order to upscale this process to industrial use. In open ponds ultrasound doesn't just have the ability to cause aggregation of the microalgae but it can also affect other sediments such as mercury (Suali and Sarbatly, 2012). At particular wavelengths ultrasound can enable the microalgae to aggregate with no shear stress at low frequency but high amplitude ultrasound has the potential to rupture the cells, releasing its contents (Kim et al., 2013). Research into biological based harvesting methods has shown that algae can flocculate spontaneously without the use of chemicals. Research has shown that increasing the natural nutrients plants use to flocculate, can increase the flocculation rate up to $100 \%$ when the nutrients in the culture medium were doubled (Silva and Silva, 2007). It would be incredibly beneficial to control this natural development as it 
would be much more ecofriendly and much less costly, however further research needs to be conducted to explore all novel biological harvesting options.

\section{Drying Methods}

Once the algae have been harvested it needs to be dried before the oil can be extracted. Various drying methods such as sun drying, freeze drying, spray drying, hot air oven and drum drying are used to remove excess water from the biomass, as solvent based lipid extraction is difficult when wet biomass is used (Wen et al., 2011). Oil yield is generally much lower with wet biomass compared to lipid extraction after a dewatering stage (Suali and Sarbatly, 2012). One drying method used is solar drying which was originally designed for the drying of seaweed for the possible use as biofuel. Solar power has become a very popular source of energy over the past few years when it comes to housing and industrial use. Most marine products require a drying stage in the process to produce a quality product (Fudholi, 2014). Extraction of lipids from the harvested algal biomass is receiving a great deal of research, as lipid extraction methods that do not require a very low water content would make the harvesting process much simpler and cheaper (Leite et al., 2013). The biomass must be processed rapidly in order to avoid the biomass form spoiling due to the enzymes within the algae hydrolyzing the cellular lipids into free fatty acids (Wen et al., 2011). It is important that during the oil extraction methods the lipids do not get damaged, however the method still needs to be quick, effective and easily scalable (Pragya et al., 2013). For oil to be extracted from the algae cells, the cell needs to be lysed and the cell membranes broken. Current commercial oil extraction methods from crops such as palm oil, uses heating under high pressure before mechanical pressing. This technique requires no chemical solvents so preserves the quality of the extracted oil, 
but it does have limitations when producing biofuels from algae due to the small size of microalgal cells (Suali and Sarbatly, 2012).

Supercritical $\mathrm{CO}_{2}$ is one method of effective oil extraction that is non-toxic, has a low critical temperature, easy separation after extraction and a high diffusivity and low surface tension (Lam and Lee, 2012). Despite these advantages of supercritical $\mathrm{CO}_{2}$ extraction, it has high costs and safety issues which make it unsuitable for extraction of microalgae oils (Wen et al., 2011; Lam and Lee, 2012; Suali and Sarbatly, 2012).

\section{Lipid Extraction}

\subsection{Electrical Extraction}

Some extraction methods use cell disruption by ultrasound or osmotic shock but each of these methods normally uses further chemical extraction using solvents such as hexane or hexane-ethanol mixture which contaminate the remaining biomass (Mata et al., 2010; Suali and Sarbatly, 2012). One such solvent extraction method is soxhelt extraction, which uses hexane to extract lipids from algal cells. This technique can be used on its own or alongside another technique such as the oil press/expeller method. After the lipids have been initially extracted by the expeller the remaining pulp can that be extracted using cyclo-hexane which dissolves oil into itself so that the pulp can be removed (Pragya et al., 2013).

\subsection{Solvents}

Extraction of lipids from the algae cells can be done through the use of solvents, the idea with using a solvent is that non-polar organic solvents disrupt hydrophobic interactions between non-polar/ neutral lipids (Pragya et al., 2013), through shifting the $\mathrm{pH}$ of the mixture to be more alkaline it can disrupt the hydrogen bonds between polar lipids. The type of solvent used depends on the algae that are being used, though 
it should be kept in mind that using a solvent that is expensive would not be viable for large scale production. The solvent would also need to be volatile, non-toxic, non-polar and a poor extractor of other non-lipid parts of the algae cells. When Ramluckan et al., (2014) came across this issue, they used an ultraviolet light to determine the amount of chlorophyll in the extracted oil. They also used a chromatographic method to identify the quantity of useable lipids. Although these further methods solve the problems with the unwanted extracted lipids it would also make the process much more expensive and time consuming. Recently described in literature is a solvent which has both polar and non-polar properties (Suali and Sarbatly, 2012; Leite et al., 2013). This extraction method uses switchable solvents which can be changed from polar to non-polar or hydrophilic to hydrophobic when bubbled with $\mathrm{CO}_{2}$ (Suali and Sarbatly, 2012; Leite et al., 2013). This enables the solvent to enter the algal cell when miscible with water and then when switched they extract the lipid out of the cells and out of the liquid, following extraction the solvent can be easily separated from the biomass and can consequently be reused, reducing production costs. This form of extraction may be suitable to use on undried biomass but future research is required to investigate this.

\section{Lipid conversion}

From these constituent parts biodiesel is produced by transesterification of the triglycerides into esters which is the final biodiesel product and glycerol which is a byproduct, the glycerol by-product can be used as a carbon source for heterotrophic algal growth so this process produces no waste (Leite et al., 2013; Zhu et al., 2014). Biodiesel is already produced using oils seeds and the reagents used for transesterification in these processes can easily be transferred and applied to microalgae biodiesel production (Leite et al., 2013). Transesterification is thought of 
as the most useful method of converting oil to biodiesel, as the process of converting the fatty acids to biofuel have the closest characteristics to those of diesel fuel (Pragya et al., 2013). Through the process of transesterification, raw lipids extracted from algae are converted to fatty acid alkyl esters which have a lower molecular weight. A major drawback to this method however is the use of methanol to drive the reaction, this affects the cost and sustainability of the conversion method (Daroch et al., 2013). Lipids from algae may also be converted to biodiesel through a process called pyrolysis, which is a thermo-chemical technique which uses heat and a lack of oxygen to break down the lipids. The product of this process is a liquid that has similar chemical components to that of petroleum diesel (Pragya et al., 2013). The known problems with this technique are temperature, particle size, sweep gas flow rate and reactor types (Hu et al, 2013).

\section{Alternative Products}

As well as the production of biodiesel there are other possible fuels that could be created manually through the use of different processes. Wen et al., (2011) also looked into biofuel from microalgae. One such fuel that they researched was biogas, such as methane produced from anaerobic digestion, which can be used as a heat source or to supply electricity (Figure 3). They mentioned Bio-oils and syngas as products from algae through the process of thermochemical conversion, which can also be categorized as gasification, pyrolysis or thermochemical liquefaction. The end products of these processes can vary between solid, liquid and gas forms. From this we understand that biodiesel is not the only useful fuel that can be created from algae. Other potential products from microalgae include biogas, fertilizer, cosmeceuticals, nutraceuticals and pigments (Suali and Sarbatly, 2012) all of which have a much 
higher value when sold on so the production of these in a closed cycle bio-refinery concept would increase the potential of industrial scale production of biodiesel and biofuels. Bioethanol can be obtained by the fermentation of sugars which are produced by hydrolyzing the carbohydrates in microalgae cells, it is described how production of bioethanol can be performed simultaneously to biodiesel but bioethanol production is still in the preliminary research phase (Lam and Lee, 2012; Zhu et al., 2014).

\section{Concerns, Limitations and Further Thoughts}

Wen et al., (2011) discovered that it would still be too expensive to use algae as a biofuel due to the cost of oil extraction and the biodiesel production, which would make it too much to commercialize. It is also highlighted that biogas production would be much more beneficial as it is much cheaper than the production of other biofuels, and it could largely contribute to a widely used fuel source such as electricity. However biodiesel is a very important fuel source as it could be largely used to power transport vehicles, which would cause much less destruction to the environment than normal diesel/ petrol we use now. This puts across the point that new technologies need to be developed that can extract the oil from the algae but also another that can turn it into biodiesel for cheaper. Alternatively the waste products from this process could be sold as animal feed, as it is full of proteins and carbohydrates. On the other hand they also had the thought that if raw biomass was used for methane production instead of biofuel production, this would massively reduce the costs as there would be no oil extraction or production of biodiesel.

Suali and Sarbatly (2012) concluded that microalgae are feasible as a biofuel feedstock, and the production could be made cheaper by using a solvent that can be reused for the extraction of lipids from the microalgae. It would also be cheaper as 
less water is needed for the process, they can reproduce more rapidly than normal plants and they have a high lipid content. Along with microalgae being used to create an alternative fuel source, it can also be beneficial in wastewater treatment and the production of pharmaceuticals. Nutrients are an essential element of algal growth, the sustainability and costs of which depend on the source of the nutrients and the recyclability (Rogers, 2013). $\mathrm{CO}_{2}$, nitrogen and phosphorus will need to be supplemented as biofuel technology develops possibly through locating algal farms close to power plants and waste water treatment plants in order to utilize their waste products.

Zheng et al (2012) looked into the use of both phototrophic and heterotrophic growth being used together. They established that using open ponds and runways to culture algae was difficult as the algae take so long to multiply. Using other culturing methods such as PBRs is extremely costly however, creating a problem when it comes to high yield production. Their main idea was to use a two-stage process involving phototrophic and heterotrophic growth, which would take the advantage of low cost from phototrophic growth and high efficiency from heterotrophic growth. They determined that heterotrophic culturing can be used as a better process to produce seed cells from the algae in large open systems, as its productivity was much higher but had the same standard of performance. Organic waste can also be used as a feedstock for heterotrophic growth making it good for the environment. Zhu et al., (2014) also discuss the potential of a recycling system in a microalgae bio-refinery chain but doesn't consider other potential products such as cosmetics, pharmaceuticals or nutritional supplements. Suali and Sarbatly, (2012) briefly discuss the potential for pharmaceutical products that can be obtained from microalgae and 
also the possibility of combining waste water treatment plants with microalgae culture methods in order to remove and utilize waste products to the benefit of the culture system.

\section{References}

Adenle, A.A., Haslam, G.E. and Lee, L. (2013) Global assessment of research and development for algae biofuel production and its potential role for sustainable development in developing countries, Energy Policy, 61, 182-195.

Burgess, J.G. (2012) New and emerging analytical techniques for marine biotechnology, Current Opinion in Biotechnology, 23, pp. 29-33.

Chisti, Y. (2007) Biodiesel from microalgae, Biotechnology Advances, 25, 294-306.

Chisti, Y. (2013) Constraints to commercialization of algal fuels, Journal of Biotechnology, 167, 201-214.

Christenson, L. and Sims, R. (2011) Production and harvesting of microalgae for wastewater treatment, biofuels, and bioproducts, Biotechnology Advances, 29, 686702. 
Daroch, M., Geng, S. and Wang, G. (2013) Recent advances in liquid biofuel production from algal feedstocks, Applied Energy, 102, 1371-1381.

Demirbas, A. (2010) Use of algae as biofuel sources, Energy Conversion and Management, 51, 2738-2749.

Fudholi, A., Sopian, K., Othman, M.Y. and Ruslan, M.H. (2014) Energy and exergy analyses of solar drying system of red seaweed, Energy and Buildings, 68, Part A, 121-129.

Gerde, J.A., Yao, L., Lio, J., Wen, Z. and Wang, T. (2014) Microalgae flocculation: Impact of flocculant type, algae species and cell concentration, Algal Research, 3, 3035.

Hadiyanto, H., Elmore, S., Van Gerven, T. and Stankiewicz, A. (2013) Hydrodynamic evaluations in high rate algae pond (HRAP) design, Chemical Engineering Journal, 217(0), pp. 231-239.

Han, F., Huang, J., Li, Y., Wang, W., Wang, J., Fan, J. and Shen, G. (2012) Enhancement of microalgal biomass and lipid productivities by a model of photoautotrophic culture with heterotrophic cells as seed, Bioresource Technology, $118,431-437$. 
Hu, Z., Zheng, Y., Yan, F., Xiao, B. and Liu, S. (2013) Bio-oil production through pyrolysis of blue-green algae blooms (BGAB): Product distribution and bio-oil characterization, Energy, 52, 119-125.

Jorquera, O., Kiperstok, A., Sales, E.A., Embiruçu, M. and Ghirardi, M.L. (2010) Comparative energy life-cycle analyses of microalgal biomass production in open ponds and photobioreactors, Bioresource Technology, 101,1406-1413.

Kim, J., Yoo, G., Lee, H., Lim, J., Kim, K., Kim, C.W., Park, M.S. and Yang, J. (2013) Methods of downstream processing for the production of biodiesel from microalgae, Biotechnology Advances, 31, 862-876.

Knuckey, R.M., Brown, M.R., Robert, R. and Frampton, D.M.F. (2006) Production of microalgal concentrates by flocculation and their assessment as aquaculture feeds, Aquacultural Engineering, 35, 300-313.

Lam, M.K. and Lee, K.T. (2012) 'Microalgae biofuels: A critical review of issues, problems and the way forward', Biotechnology Advances, 30(3), pp. 673-690.

Leite, G.B., Abdelaziz, A.E.M. and Hallenbeck, P.C. (2013) 'Algal biofuels: Challenges and opportunities', Bioresource Technology, 145, pp. 134-141. 
Li, T., Zheng, Y., Yu, L. and Chen, S. (2013) 'High productivity cultivation of a heatresistant microalga Chlorella sorokiniana for biofuel production', Bioresource Technology, 131, pp. 60-67.

Liffman, K., Paterson, D.A., Liovic, P. and Bandopadhayay, P. (2013) 'Comparing the energy efficiency of different high rate algal raceway pond designs using computational fluid dynamics', Chemical Engineering Research and Design, 91, 221 226.

Mata, T.M., Martins, A.A. and Caetano, N.S. (2010) Microalgae for biodiesel production and other applications: A review, Renewable and Sustainable Energy Reviews, 14, 217-232.

Perez-Garcia, O., Escalante, F.M.E., de-Bashan, L.E. and Bashan, Y. (2011) Heterotrophic cultures of microalgae: Metabolism and potential products, Water Research, 45, 11-36.

Pragya, N., Pandey, K.K. and Sahoo, P.K. (2013) A review on harvesting, oil extraction and biofuels production technologies from microalgae, Renewable and Sustainable Energy Reviews, 24, 159-171. 
Ramluckan, K., Moodley, K.G. and Bux, F. (2014) An evaluation of the efficacy of using selected solvents for the extraction of lipids from algal biomass by the soxhlet extraction method, Fuel, 116, 103-108.

Ritchie, R.J., Guy, K. and Philp, J.C. (2013) Policy to support marine biotechnologybased solutions to global challenges, Trends in Biotechnology, 31, 128-131.

Rogers, J.N., Rosenberg, J.N., Guzman, B.J., Oh, V.H., Mimbela, L.E., Ghassemi, A., Betenbaugh, M.J., Oyler, G.A. and Donohue, M.D. (2013) A critical analysis of paddlewheel-driven raceway ponds for algal biofuel production at commercial scales, Algal Research (Article In Press) Available online 19/12/2013.

Silva Benevides, A.M., Torzillo, Giuseppe., Kopecky, Jirka. and Masojidek, Jiri. (2013) Productivity and biochemical composition of Phaeodactylum triconutum (Bacillariophyceae) cultures grown outdoors in tubular photobioreactors and open ponds, Biomass and Bioenergy, 54, 115-122.

Silva, P.G. and Silva, H.J. (2007) Effect of mineral nutrients on cell growth and selfflocculation of Tolypothrix tenuis for the production of a biofertilizer, Bioresource Technology, 98(3), pp. 607-611.

Tang, C., and Lewis, J. (2012). Algae: Green Gold. CPI expert seminar report. P-36 
Suali, E. and Sarbatly, R. (2012) Conversion of microalgae to biofuel', Renewable and Sustainable Energy Reviews, 16, 4316-4342.

Wen, Z., Liu, J. and Chen, F. (2011) Biofuel from Microalgae, in Moo-Young, M. Comprehensive Biotechnology (Second Edition). Burlington: Academic Press, pp. 127-133.

Zheng, Y., Chi, Z., Lucker, B. and Chen, S. (2012) Two-stage heterotrophic and phototrophic culture strategy for algal biomass and lipid production, Bioresource Technology, 103, 484-488.

Zhu, L.D., Hiltunen, E., Antila, E., Zhong, J.J., Yuan, Z.H. and Wang, Z.M. (2014) Microalgal biofuels: Flexible bioenergies for sustainable development, Renewable and Sustainable Energy Reviews, 30, 1035-1046. 


\section{A. Phototrophic growth}

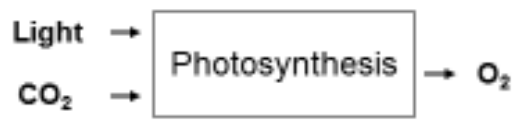

- Light

- Captures $\mathrm{CO}_{2}$

- No organic carbon

needed

- Low biomass

-Slow growth

-Low lipida content

\section{B. Heterotrophic growth}

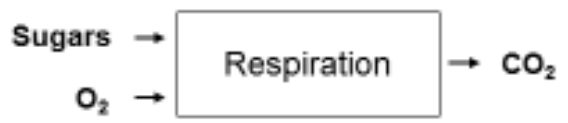

-Dark

- Emits $\mathrm{CO}_{2}$

- Organic carbon

needed

-High biomass

-Fast growth

- High lipid content

Figure1. Advartages of phototrophic and heterotrophic algal culbvation (Tang and Lewis, 2012)

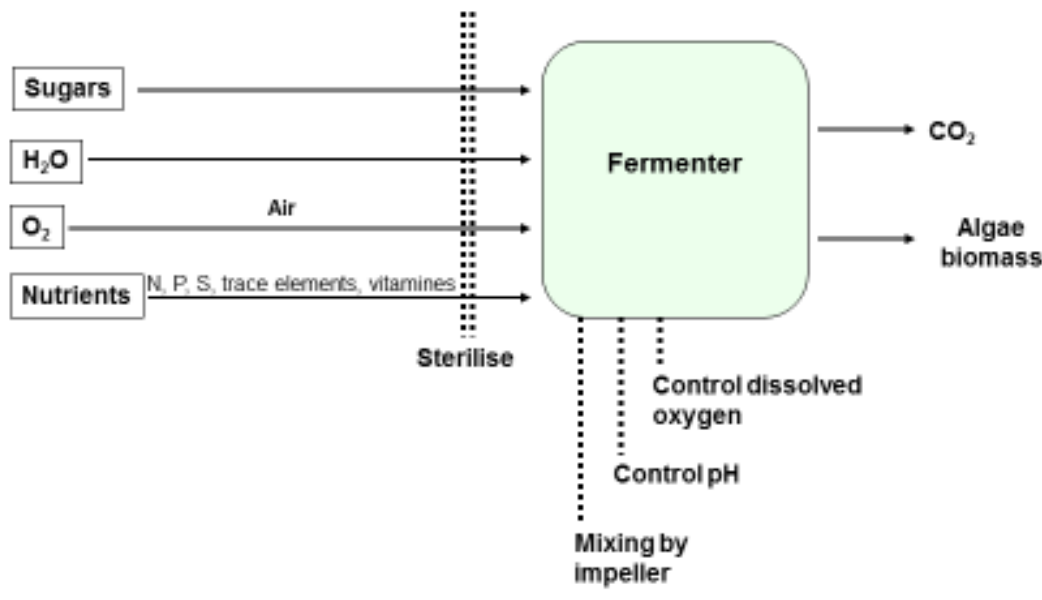

Figure 2. Algal cultivation in Heterotrophic (dark) system (Tang and Lewis, 2012) 


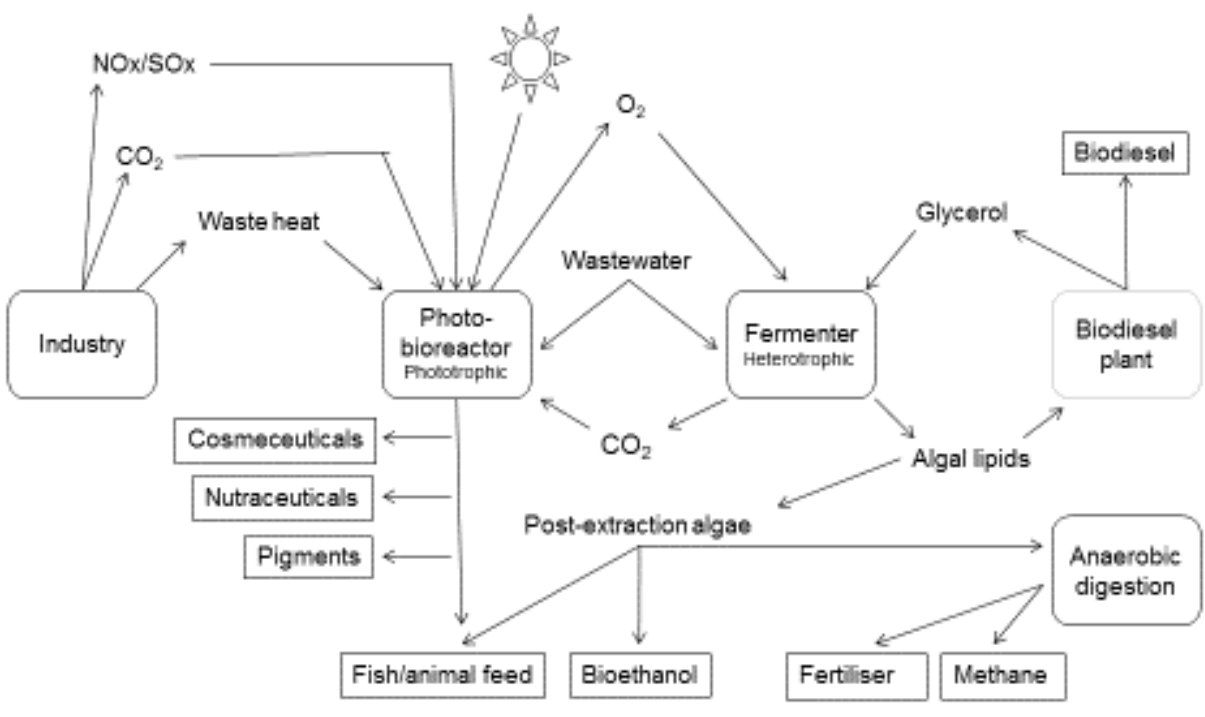

Figure 3. Biorefining and closed cyele concept (Tang and Lewis, 2012) 